\title{
Schur Convexity and the Dual Simpson's Formula
}

\author{
Yaowen Li \\ Department of Mathematics, Nanjing University, Nanjing, China \\ Email: lieyauvn@263.net
}

Received 20 December 2015; accepted 6 April 2016; published 13 April 2016

\section{Abstract}

In this paper, we show that some functions related to the dual Simpson's formula and BullenSimpson's formula are Schur-convex provided that $f$ is four-convex. These results should be compared to that of Simpson's formula in Applied Math. Lett. (24) (2011), 1565-1568.

\section{Keywords}

\section{Schur Convexity, 4-Convex Function, Dual Simpson's Formula, Bullen-Simpson's Formula}

\section{Introduction}

Schur convexity is an important notion in the theory of convex functions, which were introduced by Schur in 1923 ([1] [2]), its definition is stated in what follows. Let $R_{\geq}^{n}$ be denoted as,

$$
R_{\geq}^{n}=\left\{x=\left(x_{1}, x_{2}, \cdots, x_{n}\right) \in R^{n} ; x_{1} \geq x_{2} \geq \cdots \geq x_{n}\right\},
$$

and $\left(R_{\geq}^{n}\right)^{+}$be defined by,

$$
\left(R_{\geq}^{n}\right)^{+}=\left\{y \in R^{n} ; \sum_{i=1}^{j} y_{i} \geq 0 \text { for all } j=1,2, \cdots, n-1 \text { and } \sum_{i=1}^{n} y_{i}=0\right\} .
$$

Then we recall (see, e.g., [3]-[5]) that a function $\mathrm{f}: R^{n} \rightarrow R$ is Schur convex if

$$
\forall x, y \in R_{\geq}^{n} ; y-x \in\left(R_{\geq}^{n}\right)^{+} f(x) \leq f(y) .
$$

Every Schur-convex function $\mathrm{f}: \mathrm{D} \in R^{n} \rightarrow R$ is a symmetric function, and if $I$ is an open interval and $\mathrm{f}: I^{n} \rightarrow R$ is symmetric and of class $C^{1}$, then $\mathrm{f}$ is Schur-convex if and only if

$$
\left(x_{i}-x_{j}\right)\left(\frac{\partial f}{\partial x_{i}}-\frac{\partial f}{\partial x_{j}}\right) \geq 0, \text { on } I^{n}
$$

for all $i, j \in\{1,2, \cdots, n\}$.

Let $\mathrm{f}: I \subseteq \mathrm{R} \rightarrow \mathrm{R}$ be a convex function defined on the interval $I$ of real numbers and a, b $\in I$ with $a<b$. The following inequality 


$$
f\left(\frac{a+b}{2}\right) \leq \frac{1}{b-a} \int_{a}^{b} f(x) d x \leq \frac{f(a)+f(b)}{2}
$$

holds. This double inequality is called Hermite-Hadamard inequality for convex functions. Hermite-Hadamard inequality is improved though Schur convexity, c.f., [6]-[10]. Among these paper, it is proven that if $I \in \mathrm{R}$ is an interval and $f: I \rightarrow R$ is continuous, then $f$ is convex if and only if the mapping

$$
S_{1}(a, b)=\frac{1}{b-a} \int_{a}^{b} f(x) d x, \text { if } b \neq a
$$

(Here and what follows, we use the mapping convention $S_{\mathrm{i}}(\mathrm{a}, \mathrm{a})=\lim _{\mathrm{b} \rightarrow \mathrm{a}} S_{i}(\mathrm{a}, \mathrm{b})$ for $\mathrm{b}=\mathrm{a}$ case, which is no longer stated.) is Schur convex, and in this case, $S_{1}(\mathrm{a}, \mathrm{b})$ is convex. If $I \in \mathrm{R}$ is an interval and $\mathrm{f}: \mathrm{I} \rightarrow \mathrm{R}$ is continuous, then $f$ is convex if and only if one of the following mappings

$$
\begin{gathered}
S_{2}(a, b)=\frac{1}{b-a} \int_{a}^{b} f(x) d x-f\left(\frac{a+b}{2}\right), \quad \text { if } b \neq a, \\
S_{3}(a, b)=\frac{f(a)+f(b)}{2}-\frac{1}{b-a} \int_{a}^{b} f(x) d x, \quad \text { if } b \neq a
\end{gathered}
$$

is Schur convex. Some exciting results on Schur's majorization inequality can be found in [11]-[13].

Let $\mathrm{f}:[\mathrm{a}, \mathrm{b}] \rightarrow \mathrm{R}$ be a four times continuously differentiable mapping on [a, b]. Then the following quadrature rule is well-known:

$$
\frac{1}{b-a} \int_{a}^{b} f(x) d x=\frac{1}{6}\left[f(a)+f(b)+4 f\left(\frac{a+b}{2}\right)\right]-\frac{1}{2880} f^{(4)}(\xi)(b-a)^{4}, \xi \in(a, b),
$$

which is called Simpson's formula, c.f. [14] and [15]. For $I \in \mathrm{R}$ is an interval and $\mathrm{f}: \mathrm{I} \rightarrow \mathrm{R}$ is called fourconvex, if $f^{(4)}(\mathrm{t}) \geq 0$, for all $\mathrm{t} \in[\mathrm{a}, \mathrm{b}]$. In [15], the authors proved that if $f^{(4)}: I \rightarrow \mathrm{R}$ is continuous, then $\mathrm{f}$ is four-convex is equivalent to the mappings defined by

$$
S_{4}(a, b)=\frac{1}{6}\left[f(a)+f(b)+4 f\left(\frac{a+b}{2}\right)\right]-\frac{1}{b-a} \int_{a}^{b} f(x) d x, \text { if } b \neq a
$$

is Schur-convex, this is an improvement of the Simpson's formula.

On the other hand, the dual Simpson's formula ([14]) is stated as follows: if $f^{(4)}$ is continuous, there exist $\eta \in(\mathrm{a}, \mathrm{b})$ such that

$$
\frac{1}{b-a} \int_{a}^{b} f(x) d x=\frac{1}{3}\left[2 f\left(\frac{3 a+b}{4}\right)+2 f\left(\frac{a+3 b}{4}\right)-f\left(\frac{a+b}{2}\right)\right]+\frac{1}{23040} f^{(4)}(\eta)(b-a)^{4}, \eta \in(a, b) .
$$

In [16], Bullen proved that, if $\mathrm{f}$ is four-convex, then the dual Simpson's quadrature formula is more accurate than Simpson's formula. That is, it holds that

$$
\frac{1}{b-a} \int_{a}^{b} f(x) d x \leq \frac{1}{12}\left[f(a)+4 f\left(\frac{3 a+b}{4}\right)+2 f\left(\frac{a+b}{2}\right)+4 f\left(\frac{a+3 b}{4}\right)+f(b)\right],
$$

provided that $\mathrm{f}$ is four-convex.

Now we can state our main results. In view of the dual Simpson's formula and the above Bullen-Simpson formula, we construct two mappings as follows: for $b \neq a$, we set

$$
\begin{gathered}
S_{5}(a, b)=\frac{1}{b-a} \int_{a}^{b} f(x) d x-\frac{1}{3}\left[2 f\left(\frac{3 a+b}{4}\right)+2 f\left(\frac{a+3 b}{4}\right)-f\left(\frac{a+b}{2}\right)\right], \\
S_{6}(a, b)=\frac{1}{12}\left[f(a)+4 f\left(\frac{3 a+b}{4}\right)+2 f\left(\frac{a+b}{2}\right)+4 f\left(\frac{a+3 b}{4}\right)+f(b)\right]-\frac{1}{b-a} \int_{a}^{b} f(x) d x .
\end{gathered}
$$


We shall show that if $f^{(4)}: I \rightarrow \mathrm{R}$ is continuous, then $\mathrm{f}$ is four-convex if and only if the mapping $S_{5}(a, b)$ or $S_{6}(a, b)$ is Schur-convex. Obviously our results improve the dual-Simpson's formula and the BullenSimpson's formula, and hence complement the main result in [15].

\section{Main Results}

We now present our main theorem.

Theorem 2.1. Let $I \subseteq \mathrm{R}, \mathrm{f} \in \mathrm{C}^{4}(I)$ be a mapping on $I$, then the following statements are equivalent:

(a) The function $S_{4}(a, b)$ is Schur-convex on $I^{2}$.

(b) The function $S_{5}(a, b)$ is Schur-convex on $I^{2}$.

(c) The function $S_{6}(a, b)$ is Schur-convex on $I^{2}$.

(d) For any a, $\mathrm{b} \in I$ with $\mathrm{a}<b$, we have the Simpson inequality holds, i.e.:

$$
\frac{1}{b-a} \int_{a}^{b} f(x) d x \leq \frac{1}{6}\left[f(a)+f(b)+4 f\left(\frac{a+b}{2}\right)\right] .
$$

(e) For any $\mathrm{a}, \mathrm{b} \in I$ with $\mathrm{a}<b$, we have the dual Simpson inequality holds, i.e.:

$$
\frac{1}{3}\left[2 f\left(\frac{3 a+b}{4}\right)+2 f\left(\frac{a+3 b}{4}\right)-f\left(\frac{a+b}{2}\right)\right] \leq \frac{1}{b-a} \int_{a}^{b} f(x) d x .
$$

(f) For any $\mathrm{a}, \mathrm{b} \in I$ with $\mathrm{a}<b$, we have the Bullen-Simpson inequality holds, i.e.:

$$
\frac{1}{b-a} \int_{a}^{b} f(x) d x \leq \frac{1}{12}\left[f(a)+4 f\left(\frac{3 a+b}{4}\right)+2 f\left(\frac{a+b}{2}\right)+4 f\left(\frac{a+3 b}{4}\right)+f(b)\right] .
$$

(g) The function $\mathrm{f}$ is four-convex on $I$.

Proof:

The equivalence of (a) (d) (g) was already proven in [15]. Suppose that item (g) holds, then by the definition of the function $S_{5}(a, b)$, we have

$$
\begin{gathered}
(\mathrm{b}-\mathrm{a})\left(\frac{\partial S_{5}}{\partial b}-\frac{\partial S_{5}}{\partial a}\right)=f(\mathrm{a})+f(b)-\frac{2}{b-a} \int_{a}^{b} f(x) d x-\frac{1}{3}\left[f\left(\frac{a+3 b}{4}\right)-f\left(\frac{3 a+b}{4}\right)\right](b-a) . \\
\geq f(\mathrm{a})+f(b)-\frac{1}{3}\left[f(a)+f(b)+4 f\left(\frac{a+b}{2}\right)\right]-\frac{1}{3}\left[f^{\prime}\left(\frac{a+3 b}{4}\right)-f^{\prime}\left(\frac{3 a+b}{4}\right)\right](b-a),
\end{gathered}
$$

(by Simpson's formula (1.4) and four-convexity of f) hence,

$$
\begin{aligned}
& \frac{\partial S_{5}}{\partial b}-\frac{\partial S_{5}}{\partial a}=\frac{2}{3}\left[\left(\frac{1}{b-a} \int_{\frac{a+b}{2}}^{b} f^{\prime}(x) d x-\frac{1}{2} f^{\prime}\left(\frac{a+3 b}{4}\right)\right)-\left(\frac{1}{b-a} \int_{a}^{\frac{a+b}{2}} f^{\prime}(x) d x-\frac{1}{2} f^{\prime}\left(\frac{3 a+b}{4}\right)\right)\right] \\
& =\frac{1}{3}\left[\frac{2}{b-a} \int_{a}^{\frac{a+b}{2}} f^{\prime}\left(x+\frac{b-a}{2}\right)-f^{\prime}(x) d x-\left(f^{\prime}\left(\frac{a+3 b}{4}\right)-f^{\prime}\left(\frac{3 a+b}{4}\right)\right)\right] \\
& =\frac{1}{3}\left[\frac{2}{b-a} \int_{a}^{\frac{a+b}{2}} h(x) d x-h\left(\frac{3 a+b}{4}\right)\right] .
\end{aligned}
$$

Here we denote $h(x)=f^{\prime}\left(x+\frac{b-a}{2}\right)-f^{\prime}(x)$, for $x \in\left[\mathrm{a}, \frac{a+b}{2}\right]$. Since $\mathrm{f}$ is four-convex, $h(x)$ is convex. 
Thus Hermite-Hadamard (1.2) holds for $h(x)$ in $\left[\mathrm{a}, \frac{a+b}{2}\right]$, this gives that $(\mathrm{b}-\mathrm{a})\left(\frac{\partial S_{5}}{\partial b}-\frac{\partial S_{5}}{\partial a}\right) \geq 0$, so by the criteria (1.1) $S_{5}$ is Schur-convex, item (b) is a consequence of item (g).

Now suppose that item (b) holds. Since $\left(\frac{a+b}{2}, \frac{a+b}{2}\right) \partial(\mathrm{a}, \mathrm{b})$, Schur-convexity of $S_{5}$ gives that $0=S_{5}\left(\frac{a+b}{2}, \frac{a+b}{2}\right) \leq S_{5}(a, b)$, i.e., item (e) is valid if item (b) holds.

Next we prove item (e) implies item (g). By item (e) and the dual Simpson's formula (1.6), we get

$$
0 \leq S_{5}(a, b)=\frac{1}{23040} f^{(4)}(\eta)(b-a)^{4}, \eta \in(a, b) .
$$

Since $\mathrm{f} \in \mathrm{C}^{4}(I)$, and a, b are arbitrary, it follows that $\mathrm{f}$ is four-convex. Now the equivalence of (b) (e) (g) is proven. We follow the same pattern to show the equivalence of (c) (f) (g). If item (c) holds, then $0=S_{6}\left(\frac{a+b}{2}, \frac{a+b}{2}\right) \leq S_{6}(a, b)$, i.e., item (f) is valid. Suppose that item (f) is valid. By the definitions and formulas (1.3) and (1.4), we get

$$
0 \leq 2 S_{6}(a, b)=S_{4}(a, b)-S_{5}(a, b)=\frac{1}{2880}\left(f^{(4)}(\xi)-\frac{1}{8} f^{(4)}(\eta)\right)(b-a)^{4}, \xi, \eta \in(a, b) .
$$

Since $\mathrm{f} \in \mathrm{C}^{4}(I)$, and a, b are arbitrary, item (g) follows again. It is only left to show that item (g) implies item (c). We give a lemma first.

Lemma 2.1. Let $I \subseteq \mathrm{R}, \mathrm{f} \in \mathrm{C}^{4}(I)$ be four-convex on $I$, then the following inequalities hold for any $\mathrm{a}, \mathrm{b} \in I$ with $\mathrm{b} \geq \mathrm{a}$ :

$$
\begin{aligned}
& \frac{1}{b-a} \int_{a}^{b} f(x) d x \geq f(a)+\frac{1}{6} f^{\prime}(a)(b-a)+\frac{1}{3} f^{\prime}\left(\frac{a+b}{2}\right)(b-a) . \\
& \frac{1}{b-a} \int_{a}^{b} f(x) d x \geq f(\mathrm{~b})-\frac{1}{6} f^{\prime}(b)(b-a)-\frac{1}{3} f^{\prime}\left(\frac{a+b}{2}\right)(b-a) .
\end{aligned}
$$

Proof:

We only prove the first inequality. Denote that

$$
T(\mathrm{~b}):=\int_{a}^{b} f(x) d x-\left[f(\mathrm{a})(\mathrm{b}-\mathrm{a})+\frac{1}{6} f^{\prime}(a)(b-a)^{2}+\frac{1}{3} f^{\prime}\left(\frac{a+b}{2}\right)(b-a)^{2}\right],
$$

and that $g(x)=f^{\prime \prime}(x)$, then

$$
\begin{gathered}
T(\mathrm{a})=0 ; T^{\prime}(a)=0 ; T^{\prime \prime}(a)=0 . \\
T^{\prime}(b)=f(\mathrm{~b})-f(a)-\left(\frac{1}{3} f^{\prime}(a)+\frac{2}{3} f^{\prime}\left(\frac{a+b}{2}\right)\right)(b-a)-\frac{1}{6} f^{\prime \prime}\left(\frac{a+b}{2}\right)(b-a)^{2} . \\
T^{\prime \prime}(b)=f^{\prime}(\mathrm{b})-\left(\frac{1}{3} f^{\prime}(a)+\frac{2}{3} f^{\prime}\left(\frac{a+b}{2}\right)\right)-\frac{2}{3} f^{\prime \prime}\left(\frac{a+b}{2}\right)(b-a)-\frac{1}{12} f^{\prime \prime \prime}\left(\frac{a+b}{2}\right)(b-a)^{2} \\
=\frac{2}{3} \int_{\frac{a+b}{2}}^{b} g(x) d x+\frac{1}{3} \int_{a}^{b} g(x) d x-\frac{2}{3} g\left(\frac{a+b}{2}\right)(b-a)-\frac{1}{12} g^{\prime}\left(\frac{a+b}{2}\right)(b-a)^{2} \\
=T_{1}(b)+T_{2}(b) .
\end{gathered}
$$

Here,

$$
T_{1}(b)=\frac{1}{3}\left[\int_{a}^{b} g(x) d x-g\left(\frac{a+b}{2}\right)(b-a)\right]
$$




$$
T_{2}(b)=\frac{2}{3} \int_{\frac{a+b}{2}}^{b} g(x) d x-\frac{1}{3} g\left(\frac{a+b}{2}\right)(b-a)-\frac{1}{12} g\left(\frac{a+b}{2}\right)(b-a)^{2} .
$$

From the Hermite-Hadamard inequality for convex function $g(x)$, we see that $T_{1}(b) \geq 0$. Besides, it follows from convexity of $g(x)$ that for any $x \leq y$ :

$$
g(y) \geq g(x)+g^{\prime}(x)(y-x) .
$$

Take integration w.r.t $y$, we get

$$
\int_{x}^{y} g(y) d y \geq g(x)(y-x)+\frac{1}{2} g^{\prime}(x)(y-x)^{2},
$$

applying this inequality in $\left[\frac{a+b}{2}, b\right]$, we see that $T_{2}(b) \geq 0$. It follows that $T^{\prime \prime}(b) \geq 0$ for any $\mathrm{b} \geq \mathrm{a}$, hence by (2.1) we know $T(\mathrm{~b}) \geq 0$ for any $\mathrm{b} \geq \mathrm{a}$. The second inequality in the lemma is just the first inequality with $\mathrm{b}$ $\leq$ a, we omit its proof. The lemma is proven.

Now we continue the proof of our main theorem. By the definition of $S_{6}(a, b)$, we have

$$
\begin{aligned}
& (b-a)\left(\frac{\partial S_{6}}{\partial b}-\frac{\partial S_{6}}{\partial a}\right)=\frac{2}{b-a} \int_{a}^{b} f(x) d x-[f(\mathrm{a})+f(b)] \\
& +\frac{1}{12}\left[f^{\prime}(b)-f^{\prime}(a)\right](b-a)+\frac{1}{6}\left[f^{\prime}\left(\frac{a+3 b}{4}\right)-f^{\prime}\left(\frac{3 a+b}{4}\right)\right](b-a) \\
& =K_{1}(b)+K_{2}(b),
\end{aligned}
$$

here $K_{1}(b), K_{2}(b)$ is denoted as

$$
\begin{aligned}
& K_{1}(b):=\frac{2}{b-a} \int_{a}^{\frac{a+b}{2}} f(x) d x-f(a)-\frac{1}{12} f^{\prime}(a)(b-a)-\frac{1}{6} f^{\prime}\left(\frac{3 a+b}{4}\right)(b-a) \\
& K_{2}(b):=\frac{2}{b-a} \int_{\frac{a+b}{2}}^{b} f(x) d x-f(b)+\frac{1}{12} f^{\prime}(b)(b-a)+\frac{1}{6} f^{\prime}\left(\frac{a+3 b}{4}\right)(b-a)
\end{aligned}
$$

Suppose that item (g) holds, by applying the lemma to $\mathrm{f}$ in $\left[a, \frac{a+b}{2}\right],\left[\frac{a+b}{2}, b\right]$, we get both $K_{1}, K_{2} \geq 0$, thus $(b-a)\left(\frac{\partial S_{6}}{\partial b}-\frac{\partial S_{6}}{\partial a}\right) \geq 0$, so by the criteria (1.1) $S_{6}(a, b)$ is Schur-convex, item (c) follows.

Remark 2.1. From Lemma 2.1, we add the two inequalities together to see that the following holds for fourconvex functions $\mathrm{f}$ :

$$
\int_{a}^{b} f(x) d x \geq \frac{1}{2}[f(a)+f(b)]-\frac{1}{12}\left[f^{\prime}(b)-f^{\prime}(b)\right](b-a)
$$

it is well-known, c.f., [14] or [15].

Starting from this inequality (2.2), we deduce some properties for four-convex functions. As in the above, we define a pair of mappings $S_{7}, S_{8}$ by

$$
\begin{gathered}
S_{7}(a, b)=\frac{1}{b-a} \int_{a}^{b} f(x) d x-\frac{1}{2}[f(a)+f(b)]+\frac{1}{12}\left[f^{\prime}(b)-f^{\prime}(b)\right](b-a) ; \\
S_{8}(a, b)=\frac{1}{2}[f(a)+f(b)]-\frac{1}{b-a} \int_{a}^{b} f(x) d x-\frac{1}{12} f^{\prime \prime}\left(\frac{a+b}{2}\right)(b-a)^{2} .
\end{gathered}
$$


Then we have

Theorem 2.2. Let $I \subseteq \mathrm{R}, \mathrm{f} \in \mathrm{C}^{4}(I)$ be four-convex on $I$, then the mappings $S_{7}, S_{8}$ are non-negative and Schur-convex on $I^{2}$.

Proof:

We observe that

$$
\begin{aligned}
& (b-a)\left(\frac{\partial S_{8}}{\partial b}-\frac{\partial S_{8}}{\partial a}\right)=\frac{2}{b-a} \int_{a}^{b} f(x) d x-[f(a)+f(b)] \\
& +\frac{1}{2}\left[f^{\prime}(b)-f^{\prime}(a)\right](b-a)-\frac{1}{3} f^{\prime \prime}\left(\frac{a+b}{2}\right)(b-a)^{2} \\
& \geq \frac{1}{2}\left[f^{\prime}(b)-f^{\prime}(a)\right](b-a)-\frac{1}{3} f^{\prime \prime}\left(\frac{a+b}{2}\right)(b-a)^{2} \\
& \geq 0
\end{aligned}
$$

Here inequality (2.3) is due to inequality (2.2), and inequality (2.4) is a consequence of the Hermite-Hadamard inequality for convex function $f^{\prime \prime}$, thus by the criteria (1.1) $S_{8}$ are Schur-convex on $I^{2}$. Hence we get $S_{8}(a, b) \geq S_{8}\left(\frac{a+b}{2}, \frac{a+b}{2}\right)=0$.

Since $S_{8}$ is non-negative, we observe that

$$
\begin{aligned}
& (b-a)\left(\frac{\partial S_{7}}{\partial b}-\frac{\partial S_{7}}{\partial a}\right)=-\frac{2}{b-a} \int_{a}^{b} f(x) d x+[f(a)+f(b)] \\
& -\frac{1}{3}\left[f^{\prime}(b)-f^{\prime}(a)\right](b-a)+\frac{1}{12}\left[f^{\prime \prime}(a)+f^{\prime \prime}(b)\right](b-a)^{2} \\
& \geq-\frac{1}{3}\left[f^{\prime}(b)-f^{\prime}(a)\right](b-a)+\frac{1}{12}\left[f^{\prime \prime}(a)+f^{\prime \prime}(b)+2 f^{\prime \prime}\left(\frac{a+b}{2}\right)\right](b-a)^{2} .
\end{aligned}
$$

It is shown in [7] for a convex function $g$ that the function

$$
\left.S_{9}(a, b)=\frac{1}{4}[g(a)+g(b)]+\frac{1}{2} g\left(\frac{a+b}{2}\right)-\frac{1}{b-a} \int_{a}^{b} f(x) d x \text { (if } b \neq a\right)
$$

is Schur-convex, specially we have $S_{9}(a, b) \geq 0$. We set $g=f^{\prime \prime}$, then it is convex, we see that RHS of inequality (2.5) is non-negative, so by the criteria (1.1), $S_{7}$ is Schur-convex.

Furthermore, we give a Schur-convexity theorem for the following mapping:

$$
S_{10}(a, b)=f\left(\frac{a+b}{2}\right)-\frac{1}{2}[f(a)+f(b)]+\frac{1}{12}\left[f^{\prime}(b)-f^{\prime}(b)\right](b-a)+\frac{1}{24} f^{\prime \prime}\left(\frac{a+b}{2}\right)(b-a)^{2} .
$$

Theorem 2.3. Let $I \subseteq \mathrm{R}, \mathrm{f} \in \mathrm{C}^{4}(I)$ be four-convex on $I$, then the mappings $S_{10}$ are non-negative and Schur-convex on $I^{2}$.

Proof: We observe that

$$
(b-a)\left(\frac{\partial S_{10}}{\partial b}-\frac{\partial S_{10}}{\partial a}\right)=-\frac{1}{3}\left[f^{\prime}(b)-f^{\prime}(a)\right](b-a)+\frac{1}{12}\left[f^{\prime \prime}(a)+f^{\prime \prime}(b)+2 f^{\prime \prime}\left(\frac{a+b}{2}\right)\right](b-a)^{2} .
$$

Since $S_{9}(a, b) \geq 0$ for convex function $g=f^{\prime \prime}$, as in the above, we can conclude that $S_{10}(a, b)$ are nonnegative and Schur-convex.

Remark 2.2. For smooth four-convex functions, we see that both $S_{8}$ and $S_{10}$ are non-negative and Schurconvex functions, then the sum of $S_{8}$ and $S_{10}$ is also non-negative and Schur-convex function, especially it holds that 


$$
f\left(\frac{a+b}{2}\right)+\frac{1}{24} f^{\prime \prime}\left(\frac{a+b}{2}\right)(b-a)^{2} \geq \frac{1}{b-a} \int_{a}^{b} f(x) d x
$$

Remark 2.3. For positive real numbers $x, y$, we denote the arithmetic mean, geometric mean, and logarithmic mean of $x, y$ by $A, G, L$. Applying non-negativity of $S_{7}$ and $S_{8}$ to function $f(\mathrm{t})=\mathrm{e}^{t}, \mathrm{t} \in[\ln x$, ln $y]$ then we have

$$
\frac{1}{12} G \cdot\left(\ln \frac{y}{x}\right)^{2} \leq A-L \leq \frac{1}{12} L \cdot\left(\ln \frac{y}{x}\right)^{2}
$$

\section{Acknowledgements}

The author is partially supported by the National Natural Science Foundation of China No-11071112.

\section{References}

[1] Hardy, G.H., Littlewood, J.E. and Pólya, G. (1929) Some Simple Inequalities Satisfied by Convex Functions. Messenger of Mathematics, 58, 145-152.

[2] Schur, I. (1923) Übereine Klasse von Mittelbildungenmit Anwendungen auf die Determinantentheorie. Sitzunsber. Berlin. Math. Ges, 22, 9-20.

[3] Borwein, J.M. and Lewis, A.S. (2000) Convex Analysis and Nonlinear Optimization. Theory and Examples, CMS Books in Mathematics/Ouvrages de Mathématiques de la SMC, Vol. 3, Springer-Verlag, New York.

[4] Roberts, A.W. and Varberg, D.E. (1973) Convex Functions, Pure and Applied Mathematics. Vol. 57, Academic Press, New York.

[5] Zhang, X.M. (1998) Optimization of Schur-Convex Functions. Math. Inequal. Appl., 1, 319-330. http://dx.doi.org/10.7153/mia-01-31

[6] Chu, Y., Wang, G. and Zhang, X. (2010) Schur-Convex and Hadmard Inequality. Math. Inequal. Appl., 13, 725-731.

[7] Čuljak, V., Franjić, I., Ghulam, R. and Pečarić, J. (2011) Schur-Convexity of Averages of Convex Functions. J. Inequal. Appl., Article ID: 581918. http://dx.doi.org/10.1155/2011/581918

[8] Elezović, N. and Pečarić, J. (2000) A Note on Schur-Convex Functions. Rocky Mountain J. Math., 30, 853-856. http://dx.doi.org/10.1216/rmjm/1021477248

[9] Merkle, M. (1998) Conditions for Convexity of a Derivative and Some Applications to the Gamma Function. Aequationes Math., 55, 273-280. http://dx.doi.org/10.1007/s000100050036

[10] Zhang, X. and Chu, Y. (2010) Convexity of the Integral Mean of a Convex Function. Rocky Mountain J. Math, 40, 1061-1068. http://dx.doi.org/10.1216/RMJ-2010-40-3-1061

[11] Hwang, F.K. and Rothblum, U.G. (2004/05) Partition-Optimization with Schur Convex Sumobjective Functions. SIAM J. Discrete Math, 18, 512-524. http://dx.doi.org/10.1137/S0895480198347167

[12] Marshall, A.W. and Olkin, I. (1979) Inequalities: Theory of Majorization and Its Applications. Mathematics in Science and Engineering, Vol. 143, Academic Press, New York.

[13] Steele, J.M. (2004) The Cauchy-Schwarz Master Class. An Introduction to the Art of Mathematical Inequalities. Cambridge University Press, Cambridge. http://dx.doi.org/10.1017/CBO9780511817106

[14] Dedić, Lj., Matić, M. and Pečarić, J. (2001) On Euler Trapezoid Formulae. Appl. Math. Comput, 123, 37-62. http://dx.doi.org/10.1016/S0096-3003(00)00054-0

[15] Franjić, I. and Pečarić, J. (2011) Schur-Convexity and the Simpson Formula. Applied Math. Lett, 24, 1565-1568. http://dx.doi.org/10.1016/j.aml.2011.03.047

[16] Bullen, P.S. (1978) Error Estimates for Some Elementary Quadrature Rules. Univ. Beograd. Publ. Elektrotehn. Fak., Ser. Mat. Fiz. (No. 602-623), 97-103. 